\title{
A NOTE ON DE SITTER TYPE SOLUTIONS OF EINSTEIN'S FIELD EQUATIONS
}

\author{
A. H. KLOTZ
}

(Received 7 March 1966, revised 4 August 1966)

The exact solutions of Einstein's field equations

$$
R_{\mu \nu}=\lambda g_{\mu \nu}
$$

are determined usually by some preassigned symmetry conditions. In this note we consider a particular case of the problem to what extent their form is affected by the dimensionality of the space.

In the de Sitter metric

$$
d s^{2}=\cos ^{2} \chi d t^{2}-r_{0}^{2} d \chi^{2}-r_{0}^{2} \sin ^{2} \chi\left(d \theta^{2}+\sin ^{2} \theta d \phi^{2}\right)
$$

where $r_{0}^{2}=3 / \lambda$ (ref. 1 p. 156), the line element

$$
d w^{2}=r_{0}^{2} \sin ^{2} \chi_{0}\left(d \theta^{2}+\sin ^{2} \iota^{2} d \phi^{2}\right) \text {, }
$$

represents the distance between neighbouring points on a sphere of radius $r_{0} \sin \chi_{0}$, situated at a distance $r_{0} \chi_{0}$ from the origin of the coordinate system. It is there the two dimensional section

of the world.

$$
t=\text { const., } \chi=\chi_{0}=\text { const. }
$$

If we regard now the non-integrable element $d w / r_{0} \sin \chi$ as the differential of a single coordinate, and consider the three dimensional metric

$$
d s^{2}=h(\chi) d t^{2}-r_{0}^{2} d \chi^{2}-f(\chi) d w^{2},
$$

where $h$ and $f$ are functions of $\chi$ only, we find that the only possible solution of the field equations (1) is again of de Sitter type.

Thus, any generalisations of de Sitter space-time can be obtained only by choosing different forms of $d w$.

\section{2}

The most general form of a two dimensional line element is effectively

$$
d w^{2}=d y^{2}+k(y, z) d z^{2},
$$


where $k$ is a function of the coordinates $y$ and $z$. Let us consider the metric to be of the form

$$
d s^{2}=h(x) d t^{2}-d x^{2}-f(x)\left(d y^{2}+k(y, z) d z^{2}\right) .
$$

The field equations become

$$
\begin{aligned}
& \frac{d}{d x}\left(\frac{f^{\prime}}{f}+\frac{1}{2} \frac{h^{\prime}}{h}\right)+\frac{1}{2}\left(\frac{f^{\prime}}{f}\right)^{2}+\frac{1}{4}\left(\frac{h^{\prime}}{h}\right)^{2}=-\lambda, \\
& f^{\prime \prime}+\frac{1}{2} \frac{h^{\prime} f^{\prime}}{h}+\frac{k_{y y}}{k}-\frac{1}{2} \frac{k_{y}^{2}}{k^{2}}=-2 \lambda f, \\
& h^{\prime \prime}-\frac{1}{2} \frac{h^{\prime 2}}{h}+\frac{h^{\prime} f^{\prime}}{f}=-2 \lambda h,
\end{aligned}
$$

where dashes denote differentiation w.r.t.x and

$$
k_{y}=\frac{\partial k}{\partial y}, \quad k_{y y}=\frac{\partial^{2} k}{\partial y^{2}}
$$

It follows that

$$
f^{\prime \prime}+\frac{1}{2} \frac{f^{\prime} h^{\prime}}{h}+2 \lambda f=\alpha=-\frac{k_{y y}}{k}+\frac{1}{2}\left(\frac{k_{y}}{k}\right)^{2},
$$

where $\alpha$ is a constant.

These equations admit a solution

$$
f=a^{2} \sin ^{2} x / a, \quad h=\cos ^{2} x / a, \quad a=\text { const., }
$$

which we may call the de Sitter condition, providing

$$
\lambda=3 / a^{2} \text { and } \alpha=2 .
$$

If $\alpha \neq 2$ we find that

$$
\left(\frac{d f}{d x}\right)^{2}=\beta \sqrt{ } f+2 \alpha f-\frac{4 \lambda}{3} f^{2},
$$

where $\beta$ is an arbitrary constant. When $\beta=0$, we obtain again the de Sitter world. When $\beta$ is non-zero, the equation (10) can be integrated in terms of Weierstrass' elliptic function $\mathscr{P}$ leading to a new type of solution of field equations.

We consider in more detail the case when the conditions (9) are both satisfied. Then

It follows that

$$
\frac{\partial^{2} k}{\partial y^{2}}-\frac{1}{2 k}\left(\frac{\partial k}{\partial y}\right)^{2}=-2 k
$$

$$
k=G(z) \sin ^{2}(y+H(z))
$$


where $G$ and $H$ are arbitrary functions of $z$ only. A simple change of coordinate systems allows us to write the metric in the form

$$
d s^{2}=\cos ^{2} \chi d t^{2}-r_{0}^{2} d \chi^{2}-r_{0}^{2} \sin ^{2} \chi\left(d \theta^{2}+\sin ^{2}(\theta+\psi) d \phi^{2}\right),
$$

where $\psi=\psi(\phi)$ is a function of $\phi$ only. This solution has been obtained in a somewhat different way by Wahlquist and Estabrook [2].

The surface with the induced metric

$$
d w^{2}=d \theta^{2}+\sin ^{2}(\theta+\psi) d \phi^{2}
$$

has a Gaussian curvature +1 and is therefore isomeric to a sphere ([3] p. 133). On the other hand, it possesses some curious features.

Let us consider the simplest case: $\psi=\phi$. Assuming that the surface represents a particle we obtain semething reminiscent of an internal structure. For example, interpreting $\theta, \phi$ as polar angles on $\theta=0$ we have

or integrating from 0 to $\phi$,

$$
d w= \pm \sin \phi d \phi,
$$

$$
w= \pm 2 \sin ^{2} \phi / 2 \text {. }
$$

Since, in general relativity, a disturbance of the metric (from pseudo euclidean) is associated with matter, this can only mean either a periodic accumulation of matter, or, perhaps, a measure of the internal spin. To get some picture of it, let us represent the $w, \phi$ curve in the plane polar coordinates. Then

$$
\left(\frac{d r}{d \phi}\right)^{2}+r^{2}=\sin ^{2} \phi
$$

a solution of which may be written in terms of the series

$$
r=\frac{\phi^{2}}{2}-\frac{7 \phi^{4}}{96}+\frac{31}{11620} \phi^{6}-\cdots
$$

The general problem behind the investigation outlined above is to discover the condition under which the field equations (1) posses equivalent solutions in spaces of different dimensions. It is shown that the problem can be tackled by considering a part of the metric which represents a known surface as the differential of a single coordinate. Even in the restricted case of a de Sitter metric, this method seems to lead to new solutions capable of a physical interpretation. Such solutions will be new, however, only in the large. They arise, as we have seen, by varying the symmetry requirements on the surface (spherical in our case) which has been "shrunk to a point" in the way described. 
The author wishes to express his gratitude to Professor J. L. Synge, F.R.S., Professor T. J. Willmore (Durham University), Mr. R. Johnston (of Liverpool University), and the referee for many helpful comments.

\section{Summary}

A method of deriving a class of solutions of Einstein's field equations with symmetry of de Sitter type is investigated, and the most general solution of this kind is derived.

\section{References}

[1] A. S. Eddington, The Mathematical Theory of Relativity. (Cambridge, 1924).

[2] H. D. Wahlquist and F. B. Estabrook, Rigid Motions in Einstein Spaces (preprint).

[3] S. S. Chern, Differentiable Manifolds, (Chicago, 1959).

University of Western Australia

Perth, W.A. 